\title{
El talento de los estudiantes para la ciencia. Estrategias para su estimulación en la provincia de Mayabeque
}

DOI: $\underline{\text { https://doi.org/10.33262/ap.v1i1.20 }}$

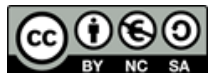

The talent of students for science. Strategies for its stimulation in the province of Mayabeque

Ariel León Arencibia., ${ }^{1}$ Beatriz de la Caridad Dorvigny González., ${ }^{2}$ \& Bárbaro Evelio Rodríguez Brito. ${ }^{3}$

\begin{abstract}
.
Talent has been studied with much more interest since the 20th century by various researchers of different approaches, developing identification and characterization models. The models proposed by Cuban specialists are static in the techniques to be used, especially in the case of the intellectual dimension where emphasis is placed on tests and tests to measure intelligence, especially the general factor (g), avoiding a more focused approach. integral. To solve the problem, the objective was raised: To develop a didactic strategy that contributes to the stimulation of academic talent in science students in the Mayabeque province. To characterize the student in the sample, the following units of analysis were defined: academic talent in adolescence and the academic talent of the pre-university student identified by their teachers. The analysis of the results was done taking into account the dimensions: intellectual, performance, creativity and social context and their indicators. The areas in which a researched talented student stands out, although it was presented as regularity in the lack of homogeneity in the dimensions and indicators evaluated.

\footnotetext{
${ }^{1}$ Universidad Agraria de la Habana” Fructuoso Rodríguez Pérez”. Facultad de Turismo. La Habana, Cuba. ariel@unah.edu.cu

${ }^{2}$ Universidad Agraria de la Habana" Fructuoso Rodríguez Pérez”. La Habana, Cuba. beatrizdg@unah.edu.cu

${ }^{3}$ Universidad Agraria de la Habana” Fructuoso Rodríguez Pérez”. La Habana, Cuba. ariel@unah.edu.cu
} 
Keywords: Academic talent, adolescent.

\section{Resumen.}

El talento se ha estudiado con mucho más interés a partir del siglo XX por diversos investigadores de distintos enfoques, desarrollando modelos de identificación y caracterización. Los modelos propuestos por especialistas cubanos son estáticos en las técnicas a emplear, sobre todo en el caso de la dimensión intelectual donde se hace hincapié en los tests y pruebas para medir la inteligencia, sobre todo el factor general (g), soslayando un enfoque más integral. Para solucionar el problema se planteó el objetivo: Elaborar una estrategia didáctica que contribuya a la estimulación del talento académico en los estudiantes para las ciencias en la provincia Mayabeque. Para caracterizar al estudiante de la muestra se definieron como unidades de análisis: el talento académico en la adolescencia y el talento académico del estudiante de preuniversitario identificado por sus profesores. El análisis de los resultados se hizo teniendo en cuenta las dimensiones: intelectual, desempeño, creatividad y contexto social y sus indicadores. Las áreas en las que se destaca un estudiante con talento investigado, aunque se presentó como una regularidad en el la carencia de homogeneidad en las dimensiones e indicadores evaluados.

Palabras claves: Talento académico, adolescente.

\section{Introducción.}

El talento académico, tema del presente artículo, se convirtió en un problema para la ciencia e inquietó a los científicos desde tiempos remotos. Existen muchas investigaciones sobre el talento académico que han implicado a diferentes ciencias como la biología, la psicología, la pedagogía, la filosofía, la sociología, la antropología y, desde finales del siglo XX, a las neurociencias. (Sánchez, 2010).

Los primeros estudios sobre el talento de corte psicológico se realizaron en Estados Unidos en los primeros cincuenta años del siglo XX por Lewis M. Terman. Él no comenzó los estudios sobre el mismo, colocándolo en el centro de sus investigaciones, sino fue a la inteligencia (Terman, 1916), luego le siguieron la solución de problemas sobre el genio y los dones en los niños (Terman \& Oden, 1947), por último se concentró en la exploración y estimulación del talento excepcional (Terman, 1954) y la medición de la inteligencia, trabajo conjunto Terman y Merrill (1959).

En el estudio de 1954, Terman introdujo el concepto de la estimulación del talento, por lo que no redujo el mismo a concepciones innatas, sino que planteó la necesidad de estimularlo, con lo que la autora intuye la complicidad de la competencia educativa en su formación y desarrollo; además llamó la atención a otros autores sobre la importancia de implicar factores personológicos y motivacionales en el desarrollo del talento.

Azuela (2004) señaló que a partir de los años setenta del siglo XX hubo gran preocupación por el talento en países como Estados Unidos, Israel y Rusia, ello estimuló la creación de centros dedicados 
a la identificación y estimulación del mismo, los más importantes según ella fueron el Center for Talented Youth y el National Center for the Talented and Gifted Research.

Por su parte, Castellanos, Vera y Vera (2005) manifestaron que otras naciones del Tercer Mundo, desde la época de los años setenta y ochenta del pasado siglo, solo han conocido un desarrollo incipiente y modesto de la identificación y estimulación del talento en las escuelas, pues otras preocupaciones han tratado de resolver, como es el caso de la calidad de la educación.

En Cuba desde finales del siglo XX se han realizado investigaciones relacionadas con el talento, las inteligencias múltiples (Correa, 2006) y las altas habilidades (Ferrándiz, Prieto, Fernández, Soto, Ferrando, \& Badía, 2010), tanto desde un enfoque pedagógico como desde la psicología educativa. Las investigaciones sobre el talento se relacionaron con la identificación y desarrollo del talento (Lorenzo R., 1996; García, 1998; Domínguez M., 2000; Vera, 2001; Castellanos, Vera, \& Vera, 2005), mientras que las investigaciones dirigidas a la determinación de características de los niños talentos en la edad escolar desde un enfoque psicológico es insuficiente.

Las investigaciones cubanas antes señaladas, según Lorenzo R (2010), fueron influenciadas por estudios realizados en las décadas de los ochentas y noventas del siglo XX, como: el Modelo Diferenciado de Superdotación y del Talento de Gagñé (1991), la Aproximación psicosocial al talento de A. J. Tannenbaum (1993), la Concepción de los Tres Anillos de Renzulli (1994), La teoría de la Inteligencia Múltiples de Gardner (1995), la Teoría Triárquica del talento de Sternberg (1997) y el Modelo Dinámico del Talento de Munich de Ziegler y Heller (Heller, 1998).

Castellanos y otros (2005) han distinguido tres grandes áreas del quehacer científico sobre el talento antes y durante el siglo XXI, ellos son: la comprensión y conceptualización del talento; la identificación y diagnóstico del talento; desarrollo y estimulación del talento. Aún, cuando en Cuba existen algunos trabajos que abordaron el problema del talento en la edad escolar desde un enfoque pedagógico en esas tres áreas (Lorenzo, 1996; García, 1998; Vera, 2001), todavía en las aulas de preuniversitario de algunas escuelas de la provincia de Mayabeque no se identifica de forma integral al estudiante de preuniversitario con talento académico, muchas veces, lo más recurrente es la tendencia a identificar solo uno de sus indicadores: el índice académico alto, por lo que se desconocen características relacionadas con dimensiones como la intelectual, el desempeño, la creatividad y el contexto social.

Estas problemáticas y otras fueron detectadas en una investigación grupal -estudio de casosdesarrollada en la provincia de Mayabeque bajo la dirección de Aroche, (2017) en el primer semestre del curso 2017 - 2018. Esta investigación constituye el antecedente del presente estudio, sus objetivos de investigación fueron: 1) Analizar cuáles métodos empíricos podrían contribuir a la identificación del talento de los adolescentes y 2) Determinar la relación que existe entre el criterio docente de identificación del talento académico en la escuela y los resultados del diagnóstico dinámico.

Las problemáticas detectadas de la presente investigación fueron:

- La mayoría de los modelos de identificación y diagnóstico propuestos por especialistas cubanos son estáticos en las técnicas a emplear, sobre todo en el caso de la dimensión intelectual donde se hace hincapié en los tests y pruebas para medir la inteligencia, sobre todo el factor general (g), soslayando un enfoque más integral. 
- En las escuelas son insuficiente las metodologías de connotación psicopedagógica para identificar el talento académico de los estudiantes en las ciencias, por lo que desconocen cuáles son y qué características presentan con vista a la estimulación del talento.

-En las escuelas son insuficientes las estrategias para el desarrollo de la creatividad en los estudiantes.

-En la formación del docente de ciencias exactas y naturales es insuficiente las asignaturas del currículo propio que propicie la estimulación del talento académico de los estudiantes

-Insuficientes cursos de posgrado por parte de la facultad de pedagogía de la UNAH para la superación permanente de los docentes que imparten las ciencias exactas y naturales en la provincia de Mayabeque

-Insuficiente explotación de las vías para estimular el talento para las ciencias de los estudiantes (aceleración, agrupamiento y enriquecimiento)

Las problemáticas antes mencionadas sirvieron de motivo para plantear como problema científico: ¿cómo contribuir a la estimulación del talento académico de los estudiantes para las ciencias en la provincia de Mayabeque?

Para resolver el problema se trazó el objetivo general: Elaborar una estrategia didáctica para la preparación del docente en la estimulación del talento académico de los estudiantes para las ciencias en la provincia de Mayabeque

En el cumplimiento del objetivo general y en la solución del problema, se plantearon los siguientes objetivos específicos:

- Analizar las concepciones teóricas y metodológicas sobre el talento en la edad escolar, sobre todo en las metodologías de tipo psicológicas para la caracterización del mismo.

- Determinar las dimensiones en las que se destacan los estudiantes talentosos y las particularidades que manifiestan en cada una.

\section{EL TALENTO EN LOS ADOLESCENTES}

En el epígrafe se le dio cumplimiento al primer objetivo de la investigación - Analizar las concepciones teóricas y metodológicas sobre el talento en los adolescentes, sobre todo en las metodologías de tipo pedagógicas para la caracterización del mismo. En el análisis teórico se examinaron las definiciones de talento por los autores que han estudiado este fenómeno y las relaciones del talento con otras áreas de la personalidad del sujeto como la creatividad y la inteligencia. Para poder caracterizar el talento de los estudiantes, es imprescindible valorar los diferentes modelos desarrollados para la identificación y caracterización del talento, así como los predictores, áreas y métodos más investigados en la edad escolar.

\subsection{El talento, acercamiento a su definición}


Para entender qué es el talento primero es necesario estudiar los antecedentes históricos y su diferencia con los conceptos de superdotado, genio, precocidad, creatividad e inteligencia, para luego responder ¿qué es el talento?

\subsubsection{Antecedentes históricos: apuntes}

El interés por conocer porqué unos seres humanos son más capaces intelectualmente que otros son tan antiguos como la humanidad. Bedia (2010) apuntó que, desde entonces, el hombre, llevado por la necesidad de resolver problemas de la vida cotidiana, "ha movilizado los procesos internos de pensamiento a la construcción de soluciones que posibilitan la adaptabilidad del individuo al medio", o sea, que la adaptación al medio ha estimulado constantemente al cerebro humano a la búsqueda de alternativas a sus problemas.

Los primeros en interesarse por el talento fueron los griegos. Platón (429-348) a partir de la correspondencia de las tres clases políticas en su Estado con las tres partes del alma humana: "la Razón (logistikón), que reside en la cabeza; el Valor (thymoeides), que se alberga en el pecho, y el Deseo (epthymetikón), que radica en el vientre..." reconoció a la virtud de la Razón como la sabiduría (sophía). (Messer, 1935, pág. 47). A partir de la concepción de quién debía gobernar a su país, Platón empleó un conjunto de pruebas para identificar el talento de los futuros gobernantes.

Según Messer (1935), Platón realizaba la identificación después de los 20 años y los dividía, según los resultados, en: a) hombres "racionales" de gran capacidad de entendimiento, los mismos eran orientados hacia el servicio de las armas; b) hombres con dotes "sensoriales" se recomendaba entrenarlos en profesiones económicas. Después de los 30 años volvía a realizar otra selección para formar al filósofo, pero solo a aquellos que habían sido instruidos durante diez años en los estudios astronómico-matemáticos. La última identificación, ocurría desde los 35 hasta los 50 años para formarlos como doctores en Filosofía. Como se puede apreciar la identificación de la capacidad para algo importante, estuvo relacionada con el desempeño de una profesión u actividad específica.

Los antiguos maestros griegos, Platón y Aristóteles (384-322), les concedieron mucha importancia a las dotes naturales (physis), su cultivo era importante para ellos. Desde la antigüedad se empleaban términos como "sabiduría", "capacidad" y "dotes naturales" propias de sujetos que las poseían y al mismo tiempo los diferenciaban de los demás.

Los términos "sabiduría", "capacidad" y "dotes naturales" permanecen en los discursos de los pedagogos humanista de los siglos XVI hasta el XVIII, los que son incluidos en orientaciones didácticas como el destacado educador alemán creador de los jardines infantiles en Alemania a principios del siglo XIX, Fröebel (1900). En su obra más conocida "La educación del hombre" usó los conceptos "talento", "inteligencia", "destrezas", "don" y "facultad creadora" como categorías fundamentales en el desarrollo del niño preescolar, y especial interés le prestó al desarrollo de la inteligencia desde las edades tempranas.

Las investigaciones psicológicas sistematizadas para identificar el talento tienen como antecedentes los estudios sobre la inteligencia, siendo los protagonistas un grupo de psicólogos nacidos en el siglo XIX, que al mismo tiempo fueron grandes pedagogos de la humanidad. 
Plancke (1959) en su estudio sobre el psicólogo, pedagogo, médico y físico belga Ovide Decroly (1871-1932) enfatizó en sus aportes a la psicología y sus intereses por las investigaciones sobre la inteligencia infantil, para lo cual se valió de observaciones y la aplicación de tests. Decroly, a partir de 1905, aplicó los test de Binet-Simon y en el proceso se da cuenta de algunas de sus debilidades como: 1) excesiva facilidad de determinadas pruebas en relación con la edad mientras que otras resultaron muy difíciles; 2) el carácter exagerado del verbalismo en el test. Estas críticas lo llevaron a realizar adaptaciones a los test y a crear otros, el más conocido es el "BD".

Uno de los primeros en escribir un texto sobre la Psicología de la Inteligencia fue el psicólogo y pedagogo ginebrino Édouard Claparède (1873-1940) consideró a la inteligencia una "facultad" primordial de la persona y la caracterizó como:

- Manera de ser los procesos psíquicos adaptados con éxitos a situaciones nuevas.

- Capacidad de resolver con el pensamiento problemas nuevos.

- Responde a una necesidad. (Claparède, 1917)

Según Venguer (1979) y Sternberg (2005a; 2005b) muchas investigaciones psicológicas del siglo XX se hicieron acompañar por "psicometristas" con fuertes intereses en las capacidades humanas. Sternberg manifestó:

(...), Francis Galton, ávido experimentalista, creó el método de correlación que fomenta los análisis psicométricos de las capacidades. Alfred Binet inventó el prototipo de test psicométrico de inteligencia más generalizado... Charles Sperman creó el análisis factorial que es la piedra angular de los análisis psicométricos de las capacidades... Edwrd Thorndike... fue el autor de un importante libro relacionado con la temática de la medición psicométrica de la inteligencia. Clark Hull, otro famoso teórico del aprendizaje, escribió su primer libro sobre el tema de tests de aptitudes, Luis Thurstone, psicometrista, era partidario de utilizar los métodos factoriales como preliminares de los experimentales, no como sustitutos. J. P. Guilford, identificado claramente con la psicometría, propuso una teoría de la inteligencia en la que una de las tres facetas que él consideraba cruciales para la inteligencia describía, justamente, los procesos de la inteligencia". (Sternberg J. R., 2005b, pág. 34)

Sternberg (2005b) reveló en este análisis que la mayoría de los estudiosos sobre inteligencia y capacidades proceden de Estados Unidos. Tanto la inteligencia como el talento para los investigadores citados por Sternberg necesitaron de la medición para determinar el desarrollo de las altas capacidades humanas. Dichos estudios se van a diferenciar por los tipos de líneas de investigación consagradas al talento, surgiendo así líderes científicos y centros dedicados al tema.

Es a partir de los años setenta del siglo XX que va a existir gran preocupación por el talento en varios países como Estados Unidos, Alemania e Israel, en muchos de ellos se crearon instituciones. Una de las instituciones destacadas fue el National Center for the Talented and Gifted Research (Centro Nacional para la Investigación del Talento y la Superdotación) dirigido por Renzulli y Sternberg como director asociado. Ambos psicólogos aportaron dos modelos para el estudio del talento los cuales serán tratados más adelante. En la universidad de Iowa, estuvo la presidencia del Consejo Mundial para la Educación de Niños Superdotados y Talentosos. 
En Europa fue Alemania el líder más poderoso en el tema del talento, no solo porque proporcionaba ayudas financieras y académicas a los alumnos con altas capacidades, sino por sus investigaciones, sobre todo desde las universidades, siendo la más destacada la Universidad de Munich con un centro de investigaciones dirigido por Heller (1998), otro importante estudioso del talento.

En Cuba los primeros estudios sobre el talento, se concentraron en el talento artístico, sobre todo el talento musical (Suárez, 1945; Díaz, 1948; Alonso, 1949). Estas investigaciones y otras que le siguieron sobre el talento, no van a tener un enfoque multidisciplinar, sino más bien pedagógico, centrado en la atención, identificación y determinación de algunas peculiaridades de niños y adolescentes con talento. Esta identificación apuntó fundamentalmente a definir a los estudiantes con:

- Altos índices académicos, más de noventa puntos, en asignaturas de matemática, física y química para entrar a los Preuniversitarios Vocacionales de Ciencias Exactas (IPVCE). (Lorenzo, 2010)

- Habilidades especiales para carreras pedagógicas y militares que se identifican mediante pruebas de concursos. (Castellanos, Vera, \& Vera, 2005)

- Habilidades específicas, detectadas por pruebas de aptitudes, para carreras de arquitectura, artes plásticas, música, danza y teatro. (Silva, 2009)

Aunque se han realizado estudios pedagógicos que proponen una identificación del talento de forma general en la edad de preuniversitario de 15-18 años (Domínguez, 2000) y escolares (Lorenzo R., 1996; Vera, 2001; Castellanos, Vera, \& Vera, 2005). Según Aroche, y otros (2012) en las escuelas de la provincia Mayabeque esta indicación está ausente debido a que no existe un conocimiento profundo sobre la identificación del talento en los profesores estudiados y además, no está estipulado como prácticas sistemáticas docentes. Estos estudios con sus fortalezas y debilidades en las concepciones de identificación y pretensiones de caracterizar, sirvieron de antecedentes para la presente investigación.

\section{Enfoque histórico cultural}

Para el autor, las concepciones sobre el talento y la creatividad del destacado psicólogo ruso de la primera mitad del siglo XX Lev Simionovich Vygotski son importantes para la identificación y caracterización del talento en la edad escolar. Vygotski le concedió mucha importancia al desarrollo de la "actividad combinadora o creadora del cerebro", sobre la misma expresó:

"Toda esta actividad del hombre cuyo resultado no es la reproducción de impresiones o acciones que formaron parte de su experiencia, sino la creación de nuevas imágenes o acciones, pertenece también a esta segunda función creadora o combinadora. El cerebro no sólo es el órgano que conserva y reproduce nuestra experiencia anterior, sino también es el órgano que combina, transforma y crea a partir de los elementos de la experiencia anterior a las nuevas ideas y la nueva conducta. Si la actividad del hombre se limitara a la reproducción de lo viejo, sería un ser volcado al pasado y sabría adaptarse al futuro únicamente en la medida en que produjera ese pasado". (Vigotski, 1987, pág. 6)

Según este autor, es en el juego donde se aprecian mejor los procesos de creación desde las más tempranas edades, es por tal razón que se incluyó en el test sociométrico la actividad de juego. 
Para la identificación y caracterización del talento no muchos autores incluyen el juego como contexto ambiental (Sternberg R. J., 2003) que propicia el desarrollo del talento mediante uno de sus indicadores: la creatividad, sin embargo, a partir de los postulados de Vygotski se tiene muy en cuenta la actividad lúdica, ya que en el juego el adolescente no revela solo "el recuerdo simple de lo vivido, sino la transformación creadora de las impresiones vividas, la combinación y organización de estas impresiones para la formación de una nueva realidad que responda a las exigencias e inclinaciones del propio niño". (Vigotski, 1987, pág. 8). Aroche (2004) manifestó que cuando un adolescente muestra un pensamiento creativo en el juego y variadas combinaciones en el contenido del juego se convierte en el líder y a los demás adolescentes les gusta jugar con él.

Otra concepción importante para la caracterización del talento en la edad escolar la aportó Vygotski, muy relacionada a la creatividad, y es la imaginación. Para él existen "cuatro formas principales que relacionan la actividad de la imaginación con la realidad"

(Vigotski, 1987, págs. 10-17): 1) "toda creación de la imaginación se estructura con elementos tomados de la realidad"; 2) relación "entre el producto terminado de la fantasía y algún fenómeno complejo de la realidad"; 3) "la relación emocional"; 4) "la estructura de la fantasía puede presentarse como algo sustancialmente nuevo". Aunque estas cuatro formas no se presentan todas en la edad escolar, las tres primeras son importantes en la caracterización del talento junto a los tipos de inteligencias: sintética (pensamiento divergente), analítica (pensamiento crítico) y práctica, planteados por Sternberg, y otros (2010).

En la provincia de Mayabeque se debe continuar avanzando en la elevación de la calidad y el rigor del proceso docente-educativo, así como en el fortalecimiento del papel del profesor frente al alumno; incrementar la eficiencia del ciclo escolar, jerarquizar la superación permanente, el enaltecimiento y atención del personal docente, el mejoramiento de las condiciones de trabajo y el perfeccionamiento del papel de la familia en la educación de niños, adolescentes y jóvenes.

Formar con calidad y rigor el personal docente que se precisa en cada provincia y municipio para dar respuesta a las necesidades de los centros educativos de los diferentes niveles de enseñanza.

Avanzar en la informatización del sistema de educación. Desarrollar los servicios en el uso de la red telemática y la tecnología educativa de forma racional, así como la generación de contenidos digitales y audiovisuales.

Ajustar la capacidad de la red escolar y el personal docente en la educación primaria, y ampliar las capacidades de los círculos infantiles en correspondencia con el desarrollo económico, sociodemográfico y los lugares de residencia.

Lograr que las matrículas en las diferentes especialidades y carreras estén en correspondencia con el desarrollo de la economía y la sociedad, incrementar la matrícula en carreras agropecuarias, pedagógicas, tecnológicas y de ciencias básicas afines. Garantizar de conjunto con las entidades de la producción y los servicios, las organizaciones políticas, estudiantiles y de masas y con la participación de la familia, la formación vocacional y orientación profesional, desde la primaria. Continuar potenciando el reconocimiento a la labor de los técnicos medios y obreros calificados

\section{Caracterización del talento adolescente:}




\section{Estudio de casos}

Escolar: LIEB Sexo: M Edad: 15 Municipio: San José

Grado: Décimo Grado

En este capítulo se le da cumplimiento al objetivo general: Caracterizar el talento académico del estudiante para las ciencias en la provincia de Mayabeque. Para dicha caracterización se parte del análisis cualitativo de cada uno de los escolares de la muestra con la ayuda del método clínico y los resultados de otros métodos empíricos.

El talento de los escolares identificados por sus profesores realizó todas las técnicas aplicadas de forma voluntaria y con deseos de colaborar en la investigación, aunque hubo alguno que no quiso realizar alguna técnica o parte de ella. Para una mejor caracterización se exponen los resultados atendiendo a las dimensiones y sus indicadores en cada caso en particular, sin llegar a la comparación de un estudiante con el otro. A continuación, aparece un caso estudiado.

\section{Conclusiones del caso LIEB:}

EL escolar LIEB posee capacidad intelectual general superior, lo que concuerda con las descripciones sobre los talentos en la adolescencia de Renzulli (1994). Su desempeño se destaca en el área académica -en las asignaturas del grado, por lo que queda claro que su jerarquía de motivos se expresa en la voluntariedad de la conducta hacia el área académica, destacándose frases como "buenas notas", "sacamos buenas notas", siendo el compromiso con la tarea estudio la más fuerte, estimulada por los resultados académicos que obtiene en las evaluaciones sistemáticas y en concursos. No manifestó interés por otras áreas de desempeño como la artística, deportiva o tecnológica, siendo una debilidad (según sus padres) su capacidad física.

La creatividad de LIEB se caracterizó porque se apoya en un material concreto, o sea, en lo visualdirecto, así en el caso del palito de tendedera primero dibuja y dice el uso real del mismo para luego modificar su uso original (para tender ropa) pero mantener su función (sujetar). Lo notable en esta escolar es que las imágenes visual-directas no se modifican enteramente en cuanto a la función del objeto dado, continúan apoyándose en la memoria reproductiva como se verificó en el dibujo imaginativo con consigna de carácter absurda.

A partir de los dos principios de Vygotski (2006b, págs. 10-11), su creación se estructuró con elementos tomados de la realidad, conservados de experiencias anteriores; solo la combinación de la realidad del palito o la aspiradora a partir de sus usos y funciones con sus nuevos usos (recoger hormigas, collares, aretes, etc.) expresan las huellas de la fantasía de la escolar más alejada de la realidad.

Los coetáneos de su grado lo seleccionaron como el más popular -estrella sociométrica- para estudiar, jugar y pasar ratos de distracción y esparcimiento, lo que demuestra que sus relaciones con sus coetáneos son positivas, aunque expresó en la entrevista preferencias por jugar y estudiar con determinados niños. La orientación hacia el futuro aún no está definida, quizás por no existir una fuerte actividad vocacional en la familia y la escuela. 


\section{Conclusiones.}

- Las investigaciones sobre el talento se concentraron fundamentalmente en los países desarrollados, en muchas de ellas se emplearon las categorías superdotado, genio, precocidad, creatividad e inteligencia como sinónimo o formando parte de la definición de la categoría talento, provocando la no existencia de una definición clara sobre dicha categoría y aceptada de forma unánime por la comunidad científica.

- Para la identificación y caracterización del talento desde un enfoque psicopedagógico existen diversos modelos con propuestas de métodos e indicadores relacionados con la inteligencia o capacidad del cerebro, el compromiso con la tarea, la creatividad, la motivación extrínseca e intrínseca y los contextos ambientales favorecedores, destructores o retardadores del talento.

- Existen escasas investigaciones relacionadas con la caracterización del talento desde las edades escolares, sin embargo, se propone por la comunidad científica especialista en el tema, la necesidad de identificar y caracterizar desde la edad escolar el estudiante con potencialidades para ser talentoso con vista a su desarrollo temprano en el área de las ciencias.

- Las áreas en las que se destacaron los escolares con talento investigados no fueron en todos los casos la misma, aunque se presentó como una regularidad en ellos la carencia de homogeneidad en las dimensiones e indicadores evaluados.

\section{Referencias bibliográficas.}

Alegría, I., Lay, S.-1., Calderón, C., \& Cárdenas, M. (2010). El proceso de construcción y validación de la Escala de Identificación de Talento Académico "EDITA". Estudios Pedagógicos, XXXVI (2), 25-39.

Allport, G. (1971). La personalidad. Su configuración y desarrollo. La Habana: Revolucionaria. Instituto Cubano del Libro.

Allport, G. W. (2002). Examen de los métodos I y II. En A. Alonso, R. Roja, \& E. Cairo, Selección de Lecturas. Diagnóstico Psicológico (págs. 48-94). Ciudad de la Habana: Facultad de Psicología. Universidad de la Habana.

Alonso, A., Cairo, E., \& Rojas, R. (2007). Psicodiagnóstico. Selección de Lecturas. Ciudad de La Habana: Félix Varela.

Amador, A. (1995). El adolescente cubano. La Habana: Pueblo y Educación.

Aroche, A. (1 al 5 de febrero de 1999). Educación artística: investigación, experiencia y soluciones. Congreso Internacional Pedagogía `99. Ciudad de la Habana, Cuba: MINED.

Aroche, A. (2004). El juego: sinfonía inconclusa. En A. Aroche, Selección de lecturas para el taller de juego (págs. 3-13). Ciudad de La Habana: ISA: Maestría de Educación por el arte.

Aroche, A. (1995). Estudio de un conjunto de actividades integradas de apreciación y producción en el área de educación plástica en Cuba. Tesis de Doctorado. La Habana Vieja, La Habana, Cuba: MINED. ICCP. 
Aroche, A. (2012). Pintar los Versos Sencillos. XI Conferencia Internacional Antropología 2012. La Habana, Cuba: Instituto Cubano de Antropología.

Aroche, A. (2009). Proyecto Sociocultural Martí y los músicos de La Edad de Oro. VII Encuentro Internacional de Cátedras Martianas. La Habana, Cuba: Centro de Estudios Martianos.

Atenberg, J. R. (2005). 3.4. Cultura e inteligencia. En Z. Bello, \& N. Estévez, Selección de Lecturas de inteligencia humana (págs. 345-413). La Habana: Félix Varela.

Bedia, L. (2010). La superdotación y el talento: una aproximación a su desarrollo conceptual. Recuperado el 9 de enero de 2012, de Monografía.com: htpp//:www.monografias.com

Castellanos, D. (2009). Atención a la diversidad y educación para el desarrollo del talento. Ciudad de La Habana: Educación Cubana.

Castellanos, D. (2014). Talento: estrategia para su desarrollo. La Habana: Pueblo y Educación.

Castellanos, D., Vera, C., \& Vera, N. (2005). El desarrollo y educación del talento. La Habana: Academia.

Castro Díaz-Balart, F. (2002). Cuba. Amanecer del Tercer Milenio. Ciencia, Sociedad y Tecnología (compilación). Barcelona: Debate.

Claparède, E. (1917). La psychologie de líntelligence. París: Scientia.

Correa, C. (2006). Propuesta metodológica para diagnosticar inteligencias múltiples en estudiantes universitarios. Tesis de Doctorado. Cienfuegos: CEDDES.

Crawford, R. P. (1954). Techniques of creative thinking. New York: Hawtorn Books. talent. En R. Sternberg, \& J. E. Davinson, Conceptions of giftedness. Cambridge: Cambridge University Press.

De Bono, E. (1974). El pensamiento lateral. Manual de creatividad. Barcelona: Paidos.

Domínguez, L. (2007). Psicología del desarrollo. Problemas, principios y categorías. La Habana: Félix Varela.

Fariñas, G. (2006). Psicología y educación de la creatividad. En G. Fariñas, Psicología, educación y sociedad (pág. 34). La Habana: Félix Varela.

Fernández, C. (1929). Plan General para la Enseñanza de Kindergarten. La Habana:

Secretaria de Instrucción Pública y Bellas Artes.

Lorenzo, R. (2013). Talento, creatividad, empresa. La Habana: Academia.

Lorenzo, R., \& Martínez, M. (1999). Talento para la ciencia: estrategia para su desarrollo. La Habana: Academia.

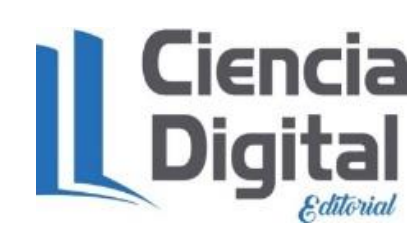




\section{Para citar el artículo indexado}

León Arencibia, A., Dorvigny González, B. de la C., \& Rodríguez Brito, B. E. (2020). El talento de los estudiantes para la ciencia. Estrategias para su estimulación en la provincia de Mayabeque. AlfaPublicaciones, 1(1), 65-76. https://doi.org/10.33262/ap.v1i1.20

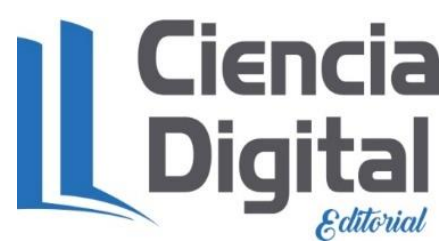

El artículo que se publica es de exclusiva responsabilidad de los autores y no necesariamente reflejan el pensamiento de la Revista Alpha Publicaciones.

El artículo queda en propiedad de la revista y, por tanto, su publicación parcial y/o total en otro medio tiene que ser autorizado por el director de la Revista Alpha Publicaciones.
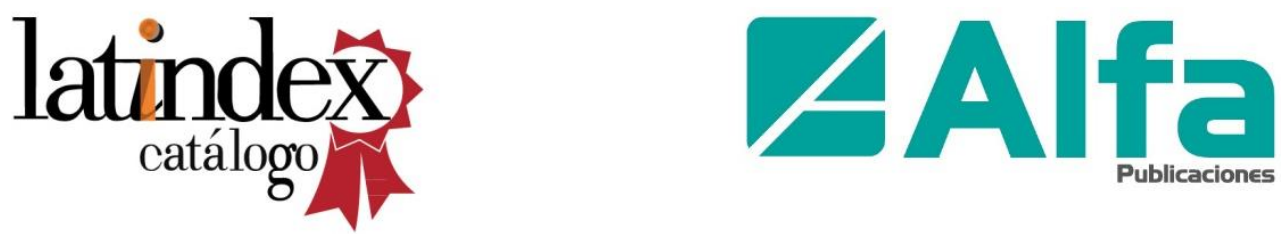